\section{Thymectomie et infection virale chez l'homme}

\section{Arguments pour un rôle du thymus à l'âge adulte}

Delphine Sauce, Victor Appay
Inserm UMRS-945, Infections et immunité, groupe Avenir, Université Pierre et Marie Curie Paris6, Hôpital Pitié-Salpêtrière, 91, boulevard de l'Hôpital, 75013 Paris, France.

delphine.sauce@upmc.fr

le nouveau-né où il occupe un volume important. La chirurgie à cœur ouvert chez les nouveau-nés est couramment pratiquée depuis vingt à trente ans, et aucun signe de déficit immunitaire (par exemple une incidence plus élevée d'infections) n'a été rapporté chez les patients thymectomisés après leur intervention.

Les jeunes adultes thymectomisés à la naissance représentent un groupe particulièrement informatif pour étudier les conséquences à long terme d'une thymectomie précoce et ainsi évaluer l'importance du thymus au-delà de la production initiale du stock de cellules T. Des études préalables ont montré que les enfants thymectomisés avaient un nombre réduit de lymphocytes CD4 ${ }^{+}$et de TREC ( $T C R$ excision cir$c l e)^{1}$ par rapport à un groupe contrôle [3-5] et que la réduction du nombre de cellules CD4 naïves était corrélée au temps écoulé depuis la thymectomie [6]. Nos propres travaux, menés chez 25 jeunes adultes (18 à 26 ans) ayant subi une ablation complète du thymus à la naissance lors d'une intervention correctrice d'une transposition des gros vaisseaux, révèlent également des altérations immunologiques assez profondes [7].

\footnotetext{
${ }^{1}$ Cercles d'excision : lors du réarrangement des chaînes du TCR des cercles d'excision (TREC) sont produits. Ces épisomes non répliqués sont retrouvés de manière très abondante dans les populations de lymphocytes $T$ nouvellement produits. Des techniques de PCR quantitatives ont été utilisées pour quantifier ces TREC dans le thymus pour avoir une estimation de la production thymique. Ces techniques peuvent aussi quantifier les TREC sur les populations de lymphocytes T périphériques.
}

\section{La fonction thymique}

Le thymus est l'organe lymphoïde centes $T$ à partir de cellules précurseurs, les prothymocytes, dérivés des cellules souches lymphoïdes issues de la moelle est double: (1) il confère une identité de type lymphocytaire $T$ aux cellules précurseurs lors de leur différenciation cification incluent le réarrangement des des récepteurs T, l'expression de ces et la multiplication des thymocytes (2) il sélectionne le répertoire antigéà discriminer les antigènes du soi et du non-soi via une sélection par les d'histocompatibilié) qui leur sont associés, réduisant ainsi indispensable à la différenciation fonccytes $T$, étapes nécessaires à l'établiseffectives.

Le thymus a grossièrement la forme d'un papillon et se situe dans une région rieur, postérieur au sternum, entre les deux poumons et en position antérieure par rapport à la veine cave supérieure. Le thymus atteint son poids maximum (relatif au poids corporel) à la naissance, puis sa taille augmente jusqu'à la puberté. Ensuite, il subit un phénomène dant généralement associée à une thymectomie car le thymus obstrue l'accès chirurgical au cœur, en particulier chez progressif, appelé involution: sa taille tissu thymique de la glande est remplacé par du tissu adipeux chez l'adulte plus agé [1]. Lactivité thymique - mesurée matures - diminue avec l'involution, même si le thymus reste le site majeur de la différenciation lymphocytaire $T$. Comme l'involution thymique est un processus naturel et relativement précoce, le rôle du thymus dans la vie adulte (c'est-à-dire une fois produit le stock initial de lymphocytes $T$ naifs) reste

\section{athie}

L'ablation du thymus (thymectomie) est d'ailleurs couramment effectué lors d'interventions chirurgicales correctrices d'une cardiopathie congénitale (CC). Les cardiopathies congénitales sont l'une des pathologies les viron 8 pour 1000 nouveau-nés [2]; elles sont extrêmement diverses, allant terférant que peu avec la croissance de l'enfant jusqu'à la malformation grave, incompatible avec la survie d cas, une chirurgie invasive représente la solution de choix pour corriger cette anomalie et ainsi sauver la vie du jeune patient. Cette intervention est cepen- 


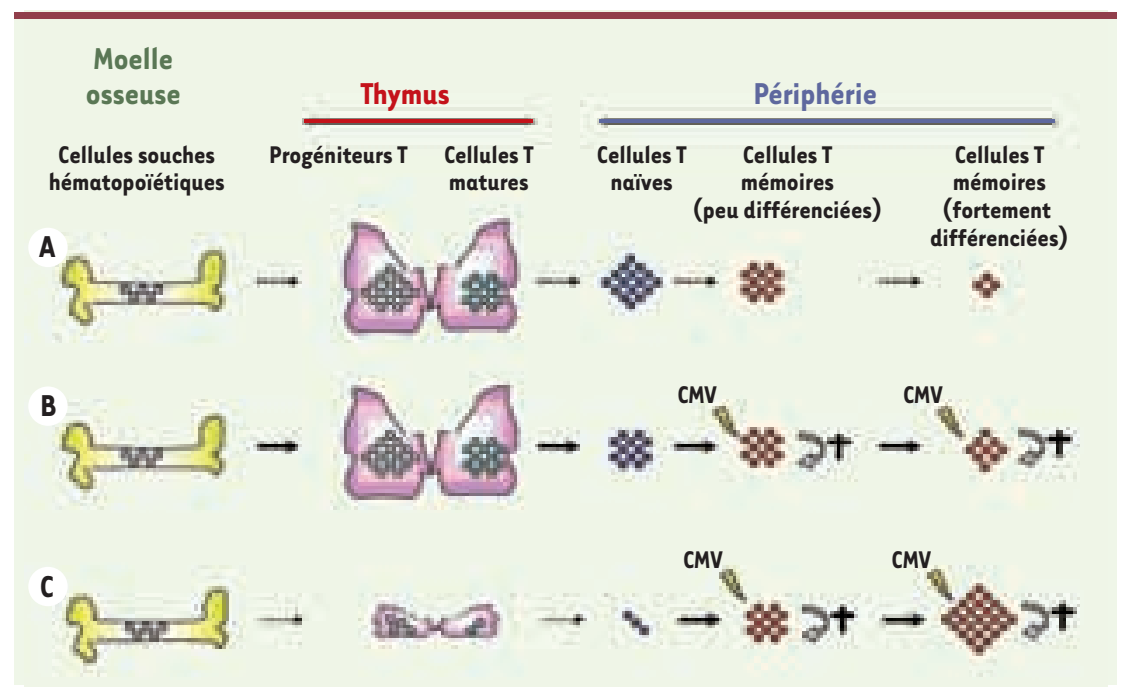

Figure 1. Représentation schématique $d u$ développement des lymphocytes T. A. Les progéniteurs hématopoïétiques (générés dans la moelle osseuse) se différencient en lymphocytes $T$ matures dans le thymus. Ces lymphocytes peuvent alors migrer vers la périphérie où ils participent à la réponse immunitaire. $\boldsymbol{B}$. La reconnaissance de pathogènes tels que le cytomégalovirus (CMV) induit l'expansion de cellules mémoires qui s'accumulent et peuvent former des populations oligoclonales fortement différenciées. Celles-ci doivent être renouvelées pour maintenir une immunité cellulaire efficace. $C$. Dans ce cas, un déficit de la capacité à renouveler le compartiment des lymphocytes T matures (dû à une thymectomie ou plus généralement à l'involution du thymus avec l'âge) conduit à une modification de l'équilibre entre les différents compartiments cellulaires de la différenciation des lymphocytes T, caractérisée par un appauvrissement des ressources en lymphocytes T et une perte de la diversité du répertoire T.

\section{Thymectomie et anomalies du système immunitaire}

Chez ces patients thymectomisés, le nombre absolu de cellules $\mathrm{CD}^{+}$est diminué, ce qui avait été décrit auparavant, mais le nombre absolu de cellules CD $8^{+}$ est également réduit. Malgré une certaine hétérogénéité parmi les donneurs étudiés, la fréquence des cellules naïves $\left(\mathrm{CD} 45 \mathrm{RA}^{+} \mathrm{CCR} 7^{+} \mathrm{CD} 27^{+}\right)$est particulièrement faible au sein des populations CD4 et $C D 8$, et cette diminution corrèle avec l'accumulation de cellules T mémoires fortement différenciées $\mathrm{CD} 57^{+}$dites sénescentes. De plus, ces patients ont un profil cytokinique particulier avec une abondance de molécules de type pro-inflammatoire, IL(interleukine)- $1 \beta$, $I L-8$, éotaxine ${ }^{2}$, dont les niveaux atteignent ceux que l'on observe chez des personnes plus âgées [7].

Malgré leur jeune âge, un sous-groupe de patients (correspondant à un tiers des patients analysés) présente des altérations particulièrement marquées au niveau du compartiment cellulaire T, équivalentes à celles de personnes âgées de plus de 75 ans: moins de $20 \%$ des

\footnotetext{
2 'éotaxine joue un rôle important dans l'inflammation allergique et chez l'asthmatique, la production bronchique d'éotaxine est accrue. Le récepteur de cette chimiokine est CCR3.
}

cellules CD4 et CD8 sont des lymphocytes naïfs et le répertoire T est biaisé (c'està-dire très oligoclonal et non polyclonal). Nous avons démontré que ce phénotype immunitaire altéré était lié à une infection par le cytomégalovirus (CMV) et en particulier à l'établissement d'une réponse immunitaire cellulaire contre ce virus. Celle-ci est mise en évidence par la mesure de la sécrétion d'IFN (interféron) $-\gamma$ en réponse aux peptides chevauchants recouvrants pp65 et IEl [7]. On sait que cet herpès virus, qui est très prévalent dans la population générale et entraîne une infection persistante de son hôte, induit une très forte expansion des cellules mémoires $T$ [8] associée à un recrutement continu du compartiment de cellules naïves [9]. L'étude des patients thymectomisés indique que l'association d'une réponse immunitaire cellulaire forte et prolongée et d'une capacité diminuée à produire de nouveaux lymphocytes peut conduire à l'épuisement prématuré des ressources lymphocytaires (Figure 1B et C). Au-delà de la production initiale du stock du lymphocytes, le thymus participe donc au maintien de l'intégrité du système immunitaire cellulaire face aux assauts répétés des pathogènes au cours de l'enfance et de la vie adulte.

\section{Vieillissement immunitaire prématuré} Les perturbations immunologiques présentées par certains patients thymectomisés, particulièrement évidentes après une infection par un virus chronique comme le CMV, apparaissent classiquement au cours de la vie, mais plus tardivement. Nos résultats indiquent que les patients thymectomisés à la naissance peuvent présenter, dès le stade de jeunes adultes, des signes prématurés de vieillissement immunitaire ou d'immunosénescence. Un nombre diminué de lymphocytes $\mathrm{CD}^{+}$et $\mathrm{CD} 8^{+}$, une proportion réduite de cellules naïves, l'accumulation de cellules mémoires oligoclonales et l'augmentation des marqueurs d'inflammation font partie des critères dits de «risque immunologique » ou immune risk profile, proposés comme marqueurs prédictifs d'une mortalité plus précoce chez les personnes âgées [10]. Il est donc possible que les patients thymectomisés développent une fragilité immunitaire précoce (par exemple caractérisée par une moins bonne réponse immunitaire contre des infections nouvelles ou après une vaccination), même si aucun ne présente actuellement de signe clinique indiquant une susceptibilité à différentes pathologies, en particulier 
infectieuses, ni n'ont démontré de sévérité augmentée lors d'épisodes infectieux. Le suivi de patients thymectomisés lorsqu'ils atteindront un âge avancé sera nécessaire pour établir si cette ablation entraîne ou non un risque plus élevé de développer des pathologies immunitaires généralement associées au vieillissement.

Comparées au bénéfice évident d'une chirurgie cardiaque néonatale pour la survie des patients souffrant de cardiopathie congénitale, les conséquences négatives potentielles de la thymectomie sur leur santé à long terme peuvent être considérées comme secondaires. Cependant, notre étude suggère qu'une ablation partielle du thymus devrait être considérée lors d'interventions chirurgicales néonatales destinées à corriger une cardiopathie congénitale, afin de préserver au maximum la capacité du tissu thymique à produire des lymphocytes au cours de la vie. $\diamond$ Thymectomy and viral infection in humans: evidence for the role of the thymus in adulthood

\section{CONFLIT D'INTÉRÊTS}

Les auteurs déclarent n'avoir aucun conflit d'intérêts concernant les données publiées dans cet article.

\section{RÉFÉRENCES}

1. Gruver AL, Hudson LL, Sempowski GD. Immunosenescence of ageing. J Pathol 2007 ; $211: 144-56$

2. Hoffman JI, Kaplan S. The incidence of congenital heart disease. J Am Coll Cardiol 2002 ; 39: 1890-900.

3. Wells WJ, Parkman R, Smogorzewska E, Barr M. Neonatal thymectomy: does it affect immune function? J Thorac Cardiovasc Surg 1998; 115: 1041-6.

4. Eysteinsdottir JH, Freysdottir J, Haraldsson A, et al. The influence of partial or total thymectomy during open heart surgery in infants on the immune function later in life. Clin Exp Immunol 2004 ; $136: 349-55$.

5. Ogle BM, West LJ, Driscoll DJ, et al. Effacing of the T cell compartment by cardiac transplantation in infancy. J Immunol 2006; 176: 1962-7.

6. Prelog M, Keller M, Geiger R, et al. Thymectomy in early childhood: Significant alterations of the CD4(+)CD45RA(+)CD62L(+) T cell compartment in later life. Clin Immunol 2009; 130 : 123-32.

7. Sauce D, Larsen M, Fastenackels $S$, et al. Evidence of premature immune aging in patients thymectomized during early childhood. J Clin Invest 2009; $119: 3070-8$.

8. Sylwester AW, Mitchell BL, Edgar JB, et al. Broadly targeted human cytomegalovirus-specific CD4+ and CD8 + T cells dominate the memory compartments of exposed subjects. J Exp Med 2005 ; 202 : 673-85.

9. Snyder CM, Cho KS, Bonnett $\varepsilon \mathrm{L}$, et al. Memory inflation during chronic viral infection is maintained by continuous production of shortlived, functional T cells. Immunity 2008 ; 29 : 650-9

10. Wikby A, Maxson P, Olsson J, et al. Changes in CD8 and CD4 lymphocyte subsets, T cell proliferation responses and non-survival in the very old: the Swedish longitudinal ОСТ0-immune study. Mech Ageing Dev 1998; 102 : 187-98.

\section{NOUVELLE}

\section{Début de spéciation chez des pinsons et des fauvettes}

Simone Gilgenkrantz
9 , rue Basse, 54330 Clérey-sur-Brénon, France. simsimone.gilgenkrantz@gmail.com
> Au cours de son voyage à bord de l'HMS' Beagle, Charles Darwin avait collecté des passeraux de diverses espèces dans les îles de l'archipel des Galapagos. À son retour, c'est en observant la taille et la forme des becs de ces oiseaux qu'il commença à réfléchir sur les variations survenues au sein d'un même groupe: «En voyant cette gradation et cette diversité de structure survenant dans un petit groupe d'oiseaux apparentés... »; il esquisse alors la première ébauche de ce qui deviendra par la suite «la théorie de l'évolution ».

Réparties dans les îles de l'archipel, plus d'une dizaine d'espèces de pinsons, désormais appelés pinsons de Darwin,

${ }^{1}$ HMS : Her Majesty's Ship sont répertoriées. Les oiseaux diffèrent en particulier par leurs becs plus ou moins grands et plus ou moins forts, selon la nature des graines dont ils s'alimentent. Les variations spontanées de la morphologie des becs ont permis que survivent et se multiplient les pinsons les mieux adaptés à la nourriture locale, et qu'à partir d'un groupe initialement identique se forment ces espèces différentes.

De même que Mendel avait judicieusement choisi Pisum sativum pour étudier la transmission des caractères, de même Darwin a été bien inspiré de s'intéresser à ces passeraux, car il semble que, chez les oiseaux, la spéciation soit facilement observable, et peut-être à des échelles de temps plus courtes qu'on le supposait, suffisamment brèves parfois pour être perceptibles au cours d'une vie d'ornithologue.

Pour preuve, deux observations d'amorce de spéciation qui viennent d'être publiées récemment, l'une sur des pinsons de Darwin (spéciation allopatrique), et l'autre sur des fauvettes à tête noire (spéciation sympatrique).

\section{Les suites d'une spéciation} allopatrique chez des pinsons de Darwin sur l'île Daphne Major Les treize espèces de pinsons vivant dans les Galapagos appartiennent à quatre genres (Geospiza, Camarhyncus, Certhidea, Pinaroloxias) et se différencient par trois traits phénotypiques: leur taille, ainsi que la forme et la taille de leur bec. L'existence de ces espèces 